\title{
Meningitis Tuberculosa
}

\author{
Dra, Patricia Vernal S.1; Dra. Teresa Param S. ${ }^{2}$; Dr. Carlos Casar C. ${ }^{3}$; Dr. Santiago Topelberg V. ${ }^{3}$
}

\section{Tuberculous Meningitis}

The clinical records of fourteen children discharged with the diagnosis of tuberculous (TB) meningitis from a metropolitan hospital at Santiago, Chile, were reviewed: Nine patients (64\% were under 5 years, but all were older than 12 month of age, eight ( $57 \%$ had positive history of exposure to TB, even though this information was not always easy to obtain and five $(36 \%)$ had BCG scars. Mycobactetium tuberculosis was isolated from the cerebrospinal fluid of 5 patients $(36 \%)$. Other four patients had miliary spread of the disease associated to the meningeal infection. The most frecuent laboratory finding was low chloride concentration in the cerebrospinal flutd (CSF). Secuelae occurret in 11 patients ( $79 \%$, consisting in bidrocephalus in ?. A clear correlation was observed between secuelae and the amount of time elapsed from admission to the begining of the specific antibacterial therapy. BCG sear does not exclude the diagnosis of TB meningitis. In patients presenting with meningoencephalic symptoms, motor involvement, cranjal nerve palsies and low CSF chloride levels the djagnosis of TB meningitis must be considered.

(Key words: Meningitis. Tubetculous. Cerebrospinal fluid chloride),

La meningitis tuberculosa (M. Tbc) es la complicación más temida de la infección primaria y causa secuelas invalidantes y muerte. En su período inicial puede tomar diferentes formas, siendo muchas veces de difícil diagnóstico especialmente en los primeros 5 años de vida. Actualmente es una enfermedad muy poco frecuente. que generalmente no se sospecha en los primeros estados $^{1,2}$, cuando sus manifestaciones son inespecificas; habitualmente el diagnóstico se retarda hasta que los pacientes han aicanzado la segunda etapa $^{3,9}$ cuando aparecen rigidez de nuca y otros signos neurológicos. Aún más, muchas veces, el diagnóstico no se realiza hasta que el paciente está en coma, o sea, en la última etapa de la enfermedad.

1. Pediatra en Estada de Perfeccionamiento, Hospital Roberto del Río.

2. Pediatra del Hospital Militar.

3. Pediatras Servicio de Pediatría del Hospital Roberto del Río:.
Debido a que el pronóstico varia según la precocidad con que se inicia el tratamiento $0^{4,5}$ es de gran importancia hacer el diagnóstico pre. cozmente e iniciar el tratamiento ante la menor sospecha.

En este trabajo se revisan los casos de $\mathbf{M}$. Tbc diagnosticados en nuestro hospital con el propósito de identificar elementos de juicio que permitan sospechar la enfermedad e iniciar el trata. miento oportunamente, reduciendo la frecuencia y gravedad de las secuelas.

\section{MATERIAL Y METODO}

Se revisaron los registros clínicos de $\mathbf{1 4}$ pacientes que egresaron del hospital Roberto del Río de Santiago con el diagnóstico de $\mathrm{M}$. Tbc. entre los meses de Enero de 1978 y Junio de 1985.

Se consignaron los siguientes datos: sexo; edad; estado nutricional; procedencia; antecedentes de enfermedad previa; contacto Tbc; vacunación BCG; reacciones con PPD; antecedentes de infección tuberculosa previa; motivo de consulta: hatlazgos en el examen físico de ingreso; diagnóstico de ingreso; t'empo transcurrido entse el ingreso y el comienzo de1 tratamiento; exáme- 
nes de laboratorio; evolución clínica; esquema de tratamiento empleado; tiempo de hospitalización $y$ secuelas al alta,

\section{RESULTADOS}

Nueve pacientes tenian entre 1 y 5 años y 5 más de 5 años (el mayor tenía $1 \mathrm{l}$ años). No hubo pacientes menores que un año. Nueve pacientes eran del sexo masculino. Diez tenían algún grado de desnutrición. No se encontraron diferencias en cuanto a procedencia urbana o rural. Se comprobaron antecedentes de contacto Tbc en 8 pacientes (57\%), pero sólo en 2 la información se obtuvo al ingreso; en los demás el antecedente se registró durante la evolución intrahospitalaria siendo en general difícil de obtener.

Se comprobó cicatriz BCG en 5 casos, pero sólo 4 habian completado el esquema de vacunación correspondiente a su edad. Entre los niños sin cicatriz BCG, en 2 el parto había ocurrido en el domicilio, otro tenía antecedente de haber nacido prematuramente, en los 6 restantes no se consignó la razón para no vacunarlos.

La reacción con PPD, efectuada durante la hospitalización, fue positiva en 5 pacientes, en 2 de ellos mayor de $15 \mathrm{~mm}$, y en uno se debió a viraje tuberculínico. E1 PPD fue negativo en otros 5 y no fue registrado o realizado en 4.

Se detectaron antecedentes de enfermedad previa en 5 pacientes: enfermedad celíaca, varicela y fiebre tifoídea en un caso cada una $y$ bronconeumonia en dos. En ningún enfermo se encontró infecçión tuberculosa previa o traumatismo encéfalocraneano.

En la Figura I se presentan los síntomas y signos más frecuentes en el momento del ingreso (los días de evolución de la enfermedad antes del ingreso fueron 8, en promedio). En la Tabla 1 se describen los síntomas y signos en relación con el tiempo de evolución de la enfermedad: los sintomas meníngeos y el compromiso de conciencia aparecen en la primera y en la segunda semana de enfermedad, en cambio el compromiso de pares craneanos y de la función motriz lo hacen más frecuentemente durante ta segunda semana de la evolución de la enfermedad.

Tabla 1.

Aparición de sintomas y signos en relación a la duración de la enfemedad. Catotce casos de meningitis $\mathrm{TBC}$.

\begin{tabular}{|c|c|c|c|c|}
\hline \multirow{2}{*}{$\begin{array}{l}\text { Síntonas } \\
\text { o signos }\end{array}$} & \multicolumn{4}{|c|}{ Días de evolución de enfermedad } \\
\hline & $0-7$ & $\overline{8-14}$ & $\overline{15-21}$ & Total \\
\hline S. meníngenos & 5 & 5 & 1 & 11 \\
\hline Convulsiones & 4 & 2 & 1 & 7 \\
\hline $\begin{array}{l}\text { Compromiso } \\
\text { conciencia }\end{array}$ & 5 & 6 & 2 & 13 \\
\hline $\begin{array}{l}\text { Compromiso } \\
\text { pares craneanos } \\
\text { Compromiso }\end{array}$ & 1 & 5 & 1 & 7 \\
\hline motor & 1 & 4 & -- & 5 \\
\hline H.I.C.* & 6 & 2 & -- & 8 \\
\hline
\end{tabular}

* Hipertensión intracraneana

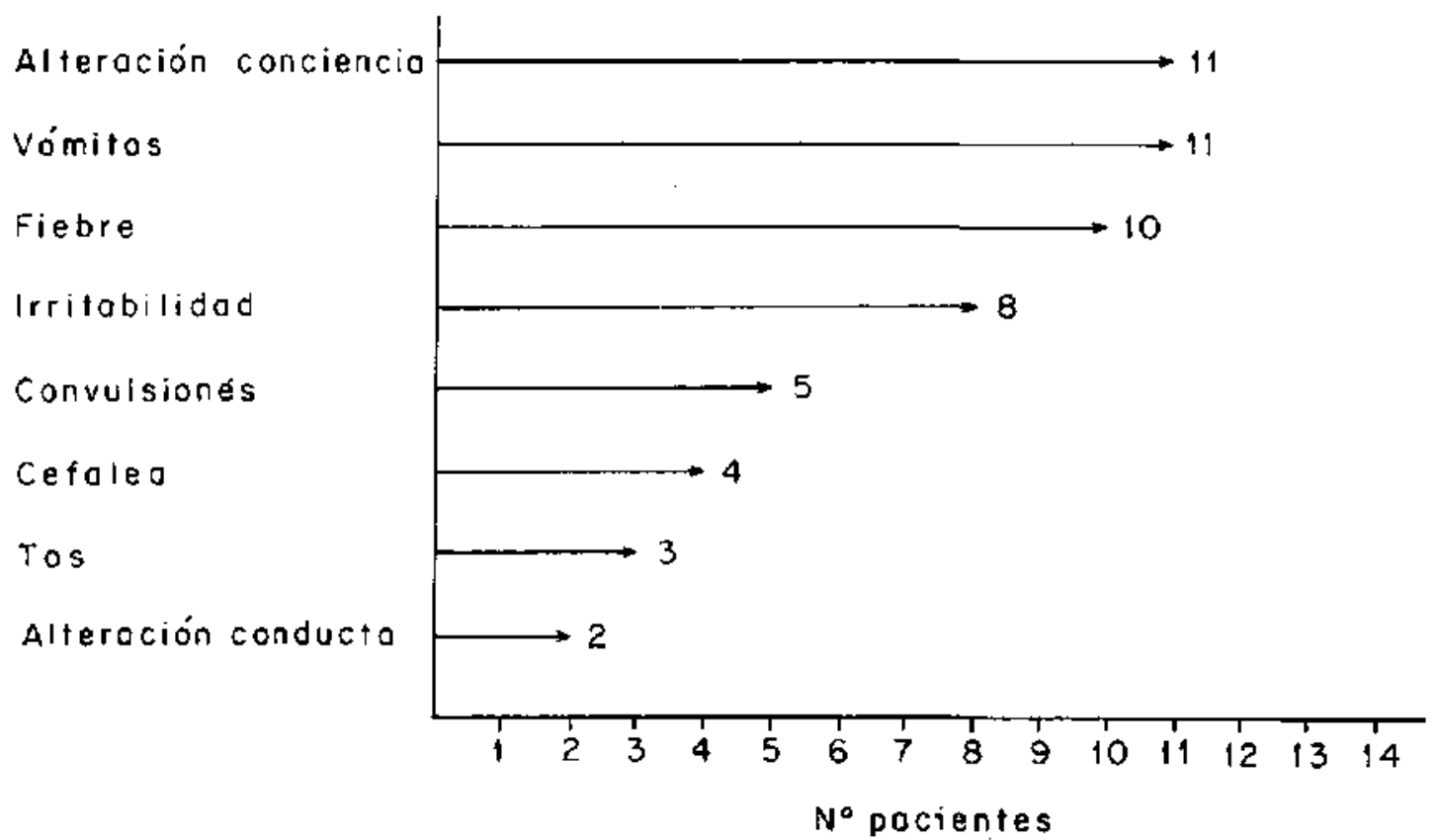

Figura I. Síntomas y signos al ingresar al hospital en 14 niños con meningitis TBC. 
Once pacientes ingresaron con alteraciones de la conciencia, 3 en coma, 2 tenían sopor, 4 sonnolencia, 2 obnubilación. En el momento de ingresar, 2 pacientes tenían compromiso de los pares craneanos, apareciendo éste en otros 5 durante la evolución. Alguros pacientes presentaron compromiso de mảs de un par. La parálisis del sćptimo par fue la más frecuentemente observada (5 pacientes), la del tercero ocurrió en 4 casos y la del sexto en uno.

El examen físico pulmonar de ingreso fue normal en 11 pacientes. En dos pacientes con examen físjco alterado se diagnosticó tuberculosis miliar (uno tenía el diagnóstico al ingreso). En 4 casos de tuberculosis pulmonar, miliar o no, el examen clínico de ingreso fue nomal. El diagnóstiço de meningoencefalitis se hizo en el momento del ingreso en 8 pacientes, en dos de ellos se sospechó la etiología tuberculosa y un tercero fue trasladado a nuestro hospital con el diagnóstico comprobado (Tabla 2).

El tiempo transcurrido entre el ingreso y la sospecha de meningitis tuberculosa fue menor de una semana en 8 casos; en un caso el diagnóstico no había sido sospechado y se hizo cuándo el cultivo del liquido cefalorraquídeo (LCR) dio resultados positivos.
El diagnóstico fue confirmado por cultivos positivos para bacilos de Koch en el LCR en 5 casos. En el resto los cultivos resultaron negatiyos: encontrándose en 4 de ellos $\mathrm{Tbc}$ muliar concomitante. No hubo confimación bacteriológica del diagnóstíco en 5 niños, en quienes se fundamentó en el sindrome clinico, de laboratorio $y$ la evolución: en 2 de estos últimos pacientes habian imágenes radiológicas pulnonares sugerentes de Tbc. En un caso se encontró meningitis por Streptococo pneumoniae concomitantemente.

En los exámenes de laboratorio el hemograma mostró evidencia de anemia en 2, leucocitosis $\left(>12.000\right.$ leucocitos por $\left.\mathrm{mm}^{3}\right)$ en $4 \mathrm{y}$ desviación a izquierda en 5 pacientes. No se encontró leucopenia. La velocidad de sedimentación globular (VHS) se encontró al terada en 7 (rảngo 30 a 70), siendo normal en 4 casos.

Todas las baciloscopias del contenido gástrico, expectoración y LCR, fueron negativas.

En la Tabla 3 se detallan las alteraciones del LCR encontradas en el ingreso. La glucosa fue normal en 2 pacientes, y bajo límites nomales en 12 ( $<40 \mathrm{mg} \%$ ). La gran mayoría de los pacientes tenian alteraciones de la concentración albúmina, en rangos variables; las concentracio-

Tabla 2.

Diagnóstico de ingreso en 14 pacientes con meningitis TBC. Hospital Roberto del Río, 1978-1985.

\begin{tabular}{|c|c|c|c|c|}
\hline Meningoencefalitis & $: 8$ & -1 & $\begin{array}{l}\text { Meningitis TBC } \\
\text { Meningitis bacteriana aguda } \\
\text { Meningitis viral }\end{array}$ & $\begin{array}{l}: 3 \\
: 4 \\
: 1\end{array}$ \\
\hline Sindrome convulsivo & $: 3$ & \{ & $\begin{array}{l}\text { + Intoxicación alcohólica } \\
\text { + Epilepsia } \\
\text { + Hemiparesia braquioctural }\end{array}$ & $\begin{array}{l}: 1 \\
: 1 \\
: 1\end{array}$ \\
\hline S. Febril prolongado & $: 2$ & & (F. Tifoidea: 1 ) & \\
\hline S. malabsorción & $: 1$ & & & \\
\hline
\end{tabular}

Tabla 3.

Alteraciones del LCR en 14 enfermos con meningitis TBC (al ingreso)

\begin{tabular}{|c|c|c|c|c|c|}
\hline & Normal & & Alterado & & Rango \\
\hline Glucosa & 2 & & 12 & & $(40 \mathrm{mg} \%)$ \\
\hline Albúmina & 2 & & 11 & & $(26-350 \mathrm{mg} \%$ \\
\hline Cloruros* & 1 & & 10 & & $(520-650 \mathrm{mg} / 1)$ \\
\hline Leucocitos & 1 & & 13 & & $\left(-2-870 \times \mathrm{mm}^{3}\right)$ \\
\hline Predominio de polinucleares** & 3 & $50 \%$ & 3 & $50 \%$ & \\
\hline Predorninio de mononucleares & 7 & $50 \%$ & 3 & $50 \%$ & \\
\hline
\end{tabular}

* En 3 casos no se consignó.

** En 4 pacientes no se consignó predominio. 
nes de cloruros se encontraron claramente disminuidas en casi todos los pacientes en que fue registrada (10/11) con rango entre 520-6.50 $m g \%$, en $1 / 11$ fue normal $y$ en $3 / 11$ no fue consignada.

Al relacionar las alteraciones del LCR con e] tiempo de evolución transcurrido desde el comienzo de la enfermedad, no se encontraron alteraciones significativamente diferentes con las ya analizadas. Durante la evolución clinica los 2 pacientes cuya glucorraquia era normal al ingresar, mostraron descensos a concentraciones inferiores a las normales en controles posteriores. E1 paciente con concentración normal de cloruros evolucionó con descenso de éstos en controles posteriores; en el paciente cuyo recuento leuco. citario fue inicialmente normal, éste aumentó posteriomente.

Se hicieron registros de electroencefalograma (EEG) en 9 pacientes siendo normal sólo en uno: en 7 hubo compromiso difuso y en uno descargas multifocales.

Las radiografías de tórax eran nomales en 6 pacientes y demostraron compromiso pulmonar atribuíble a tuberculosis en 6: miliar (4/6), complejo primario (1/6), TBC pulmonas (1/6). En otros dos niños se encontraron imágenes calificadas de neumopatias intercurrentes.

En 13 pacientes se practicó fondo de ojo, sin mostrar alteraciones.

En seis pacientes se realizaron tomografías axiales computadas (TAC) del cráneo durante la evolución de la enfermedad, en todos por sospechar alguna complicación; en 4 demostró hidrocefalia, en uno edema cerebral y resultó normal en el sexto.

Se demostró secreción inadecuada de hormona antidiurética en 3 pacientes, en los demás no se investigó o no fue consignada.

Once pacientes presentaban secuelas en el momento del alta: hidrocefalia (7/11); trastornos de la conducta $(2 / 11)$, hemisparesia y ceguera (1/11) y sindrome convulsivo (1/11).

Todos los pacientes fueron tratados con Rifampicina, Izoniazida, Estreptotomicina, Pirazinamida y corticojdes según el esquema nacional en uso. Un paciente sufrió por intolerancia a la Rifampicina presentando ictericia y aumento de la concentración de transaminasas en el suero.

El promedio de permanencia en el hospital de nuestros enfermos, fue 57 días. Posteriormente completaron su tratamiento en un Sanatorio. Ninguno de los catorce enfermos fafleció.

\section{DISCUSION}

Este estudio muestra que la meningitis tuberculosa es poco frecuente ahora en nuestro medio, predomina en menores de 5 años de edad y en niños de sexo masculino como en ottas series ${ }^{6}$. La ausencia de cicatriz BCG en la mayoría de los casos y la impresionante disminución de la incidencia de meningitis Tbc en las edades menores desde que se ha empleado en Chile la vacunación con BCG apoyan la importancia de esta medida preventiva $2,7,9$.

Concordando con la literatura, un porcentaje importante presentaba algún grado de desnutri. ción 1,7 . El antecedente de enfermedad previa en algunos pacientes es un dato digno de considerar pero no resulta concluyente.

La elevada proporción de casos en que se encontró antecedentes de contacto Tbc conocido, subraya su importancia para el diagnóstico. Es digno de mención el hecho de ser un antecedente dificil de obtener.

El hemograma no orienta hacia el diagnóstico. En el líquido cefalorraquídeo, en concordancia con otras experiencias $10,11,12$, se observa con mayor frecuencia hipoglucorraquia, albuminorraquia aumentada y predominio de células mononucleares en el recuento celular. Estas alteraciones tienen, sin embargo, amplio rango de variaciones pudiendo encontrarse glucorraquias y albuminorraquias normales ocasionalmente y, en algunos pacientes, predominio de polimotfonucleares con recuentos celulares muy variables, independientes de los dias de evolución de la enfermedad. No ocurre lo mismo con la concentración de cloruros que invariablemente se encuentra disminuida con respecto a las cifras normales.

Se destaca el hecho que el examen de fondo de ojo no mostró alteraciones en nuestros pacientes, incluso en aquellos con Tbc miliar en los cuales se describe con mayor frecuencia ta presencia de tubérculos coroídeos 1 .

La concomitancia de Tbc pulmonar fue de gran ayuda para el diagnóstico1,12,15 ya que el cultivo fue positivo sólo en $36 \%$ de nuestros pacientes lo que es comparable con otras publicaciones ${ }^{6,11}$.

Uno de nuestros pacientes con Tbc miliar y meningitis Tbc sufrió concomitantemente meningitis neumocócica, hecho que está descrito y ocurre en $11 \%$ de los casos, siendo las asociaciones más frecuentes con Staphylococcus aureus $y$ Streptococcus pneumoniae ${ }^{1}$.

El elevado porcentaje de secuelas de esta serie y las características de ellas reafirma lo descrito ${ }^{9}$ : casi todos nuestros pacientes consultaron tardiamente (etapas 2 y 3 de la enfernedad) excepto uno que ingresó en la primera etapa y evolucionó favorablemente sin secuelas. $\mathrm{La}$ iniciación de la terapia antituberculosa en los pa, cientes con secuelas demoró en promedio 18 días 
desde el comienzo de los síntomas (8 a 35 días) la que contrasta con los 3 pacientes sin secuelas al alta que tuvieron una latencia promedio de 6 dias de evolución de enfermedad antes de comen. zar el tratamiento.

El diagnóstico de meningitis es dificil en la primera etapa, salvo que curse con tuberculosis de otra Jocalización, generalmente pulmonar, por lo que frente a un caso individual conviene recordar que la presencia de cicatriz BCG no descarta el diagnóstico. Además resulta conveniente buscar antecedentes de contacto tuberculoso en forma dirigida en cualquier caso de meningitis o meningoencefalitis con LCR claro. Por lo general la reacción al PPD, no ayuda en e] diagnóstico. El diagnóstico es muy probable en los sindromes meningoencefálicos con compromiso motor, de pares craneanos o ambos. Conviene pensar en la posibilidad de la meningitis Tbc siempre que se encuentren concentraciones disminuidas de cloruro en LCR.

\section{RESUMEN}

Se revisan las observaciones clínicas de 14 pacientes con meningitis tuberculosa. Predominaron los pacientes menores de 5 años. El antecedente de contacto Tbc se comprobó en el $57 \%$, siendo en general un dato de difícil obtención y en la mayoría retrospectivo. Se observó cicatriz BCG en 5 casos. El diagnóstico se confimmó con cultivo de Koch en LCR en 5 casos ( $36 \%$ ). En otros 4 casos había Tbc pulmonar concomitante. El hallazgo de laboratorio más constante fue la concentración de cloruros del LCR, que invariablemente resultó disminuida.

Un porcentaje importante de casos sufrió secuelas (11 casos, 79\%) la mayoría fueron hidrocefalias; encontrándose clara relación entre ellas y el retardo en el comienzo del tratamiento.

La existencia de cicatriz BCG no descarta el diagnóstico de meningitis Tbc. Se debe sospechar la enfermedad en pacientes con síntomas meningoencefálicos con compromiso motor de pares craneanos o ambos y cuando la concentración de cloruros en LCR está bajo límites normales.

\section{REFERENCIAS}

1. Opitz H. Schmidt F.: Enciclopedia Pediátrica. Madrid: Ed. Morata. 1963. pp. 793.

2. Stockins B., Carrasco E.: Meningitis Tuberculosa. Bol. Hosp. San Juan de Dios 18: 427, 1971.

3. Miller F.J.W.: Tuberculosis in Children. Edimburgh: Churchil Livingstone 1982, pp, 168.

4. Idriss $Z$.: Tuberculous Meningitis in Childhood. Am. J. Dis. Child, 130: 364, 1976.

5. Kennedy D.H., Falkon R.J.: Tuberculous Meningitis. JAMA 241: 264, 1979.

6. Soza G., Ganido J., Postor P.: Meningitis Tuberculosa en la infancia. Rev. Chil. Pediatr. 48: 76, 1977.

7. Jaffe I.P.: Tuberculous Meningitis in Childhood. Lancet. 1: 738, 1982.

8. Medina E.: Estado actual del problema de la Tuberculosis en Chile. Rev. Méd. Chil 111: 323, 1983.

9. Hyarocephatus in tuberculous meningitis in children: treatment with acetazolamine and repeat lumbar puncture. J. Pediatr. 95: 657, 1979.

10. Follon R.J., Kennedy D.H.: Tuberculous Meningitis in children. Lancet. $1: 392,1982$.

11. Burdach R., Maffioletti F., Palacios E.: El liquido cefalorraquídeo en la Meningitis Tuberculosa. Pediatría (Sigo.) 171, 1963.

12. Onetto R., Weinstein M., Velasco $N, y$ cols.: Meningitis tuberculosa en Adolescentes y Adultos. Rev. Med. Valparaíso 16: 111, 1963.

13. Noughten $E$, Wendling A.M., Newton R., Bower $\boldsymbol{B} . \boldsymbol{A}$.: Tuberculous meningitis in children, Lact. 2 : 973, 1981.

14. González $M$, Vallejos $E$, Tamico A. y cols.: Electrolitos y $\mathrm{Ph}$ en el L.C.R. y en la sangre de niños con meningitis tuberculose Pediatría (Stgo.) 8: $275,1965$.

15. Schuti K.E.: Miliasy Tubercolusis in children. Am. J. Dis Child. 133: 583, 1979.

16. Parra M., Rojas P., Brinck G.: Meningitis Tuberculosa del Adulto. Experiencia clínica en el Hosp. J.J. Aguirre de la Universidad de Chile, años 1954-1966. -Rev. Chil. Neuropsiquiatr. 7: 15, 1968.

17. Swart S., Briggs R.S., Milliac P.A.: Tuberculous Meningitis in Asian patients. Lancet. 2: 15, 1981.

18. Fitzsimmons I.: Tuberculous Meningitis. Tubercle (London) 44: 87, 1981.

19. Corner B., Anderson J.D.: A family outbreck of Tuberculosis in Bristol in 1973 with two cases of Toberculous Meningitis. Tublercle 55: 99, 1974.

20. Buchinan $N$ : Radioactive Bromide partition test in the diagnosis of Tuberculous Meningitis. J. Pediatr. 99: 506,1981 .

21. Wiggelinkhwizen $f$, Mome $M$ : The radioactive bromide partition test in the diagnosis of tuberculous meningitis in children. J. Pediatr. 97: $843,1980$. 